\title{
Improved Protocol to Tackle the pH Effects on Membrane-Inserting Peptides
}

\author{
Tomás F. D. Silva, ${ }^{\dagger}$ Diogo Vila-Viçosa, ${ }^{\dagger, \dagger}$ and Miguel Machuqueiro*,† \\ †BioISI: Biosystems and Integrative Sciences Institute, Departamento de Química e \\ Bioquímica, Faculdade de Ciências, Universidade de Lisboa, 1749-016 Lisboa, Portugal \\ $\ddagger$ Kinetikos, Coimbra, Portugal \\ E-mail: machuque@ciencias.ulisboa.pt
}

Phone: +351-21-7500112

Supporting Information

April 1, 2021 


\section{List of Figures}

Figure S1: Time series plot of Asp25 residue distance relative to the membrane center for all simulated systems at pH 6.0 (352 lipids, A; 256 lipids, B; 192 lipids, C; 160 lipids, D; 128 lipids, E). This residue is known to sample the membrane center in unperturbed membrane systems. ${ }^{1}$ At $0 \AA$, the grey region represents the membrane center, while positive distance values indicate the residue is at the N-terminus membrane monolayer, while negative distance values refer to the C-terminus monolayer. Probability density distribution calculated with the previous data for every lipid membrane system (F). The time series show the overall peptide vertical movement along the simulations and the distributions depict the average peptide vertical position using Asp25 as reference. S4

Figure S2: Average helicity percentage of $w t$-pHLIP in state III obtained through DSSP analysis, at each pH value, for every lipid membrane system. The represented helicity values are a measurement of peptide folding equilibrium in the sampled conformational space. The errors bar shown were obtained by calculating the standard

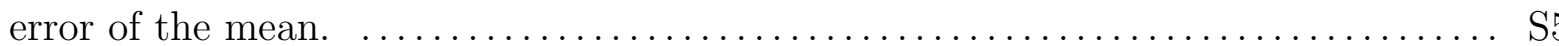

Figure S3: Helicity profiles for the Asp14 region of pHLIP (residues 12-16) at different pH values for the 256 (top left) and 352 (top right) lipid systems. A direct comparison between the two systems at pH 6.0 is also shown (bottom plot). The helicity is quantified by counting the number of residues in helical secondary structure classifications

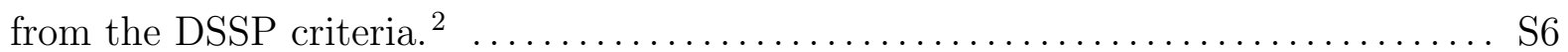

Figure S4: Water radial distribution function (RDF) from an aspartate pentapeptide in a 128 DMPC membrane bilayer. The data was extracted from previous published simulations. ${ }^{3}$ Both water oxygens were used for the calculations. The first, second, and third solvation shells are at $0.42,0.65$ and $0.85 \mathrm{~nm}$ respectively. The two peaks inside the first solvation shell appear due to established hydrogen bonds with either

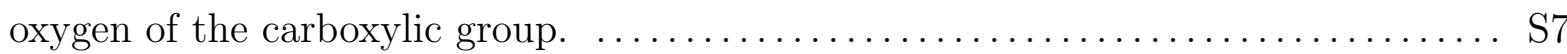


Figure S5: Minimum phosphate distance distributions for pHLIP Asp14 residue in the 256 POPC membrane bilayer. Both free oxygens of the phosphate group were used for the calculations. The interaction shell at $0.52 \mathrm{~nm}$ encapsulates all local interactions with the phosphate groups that compete with water molecules and include both direct

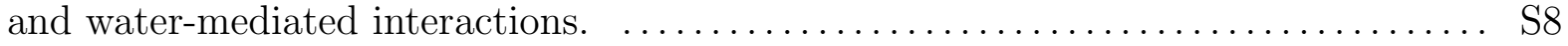
Figure S6: Protonation profiles and membrane insertion distributions of Asp14 at pH 6.0 for all simulated systems, including the one with 128 lipids. The membrane is highlighted using a grey-shaded region. The negative insertion values indicate residue insertion relative to the water/membrane interface defined by the phosphate groups $(0 \AA)$. The positive insertion values indicate that the residue is solvated by bulk water. S9

Figure S7: Time series plot of the minimum distance between the Asp14 carboxylic oxygen atoms and the free oxygen atoms of the phosphate groups. The data was obtained from two replicas of the 352 (upper plot) and 256 (bottom plot) lipid size system, illustrating two very distinct behaviours in our simulations. In replica 1 (blue dots), we observe a "lock" state behaviour, with Asp14 persistently interacting with a phosphate group most of the simulation time. Meanwhile, in replica 2 (red dots), the exhibited behaviour reveals an interchange between "on/off" states, where Asp14 is able to establish and break hydrogen bonds throughout the simulation. The grey shaded region highlights the range of distances (below $0.3 \mathrm{~nm}$ ) defining a stable hydrogen bond between Asp14 and a phosphate group. .................. S10 

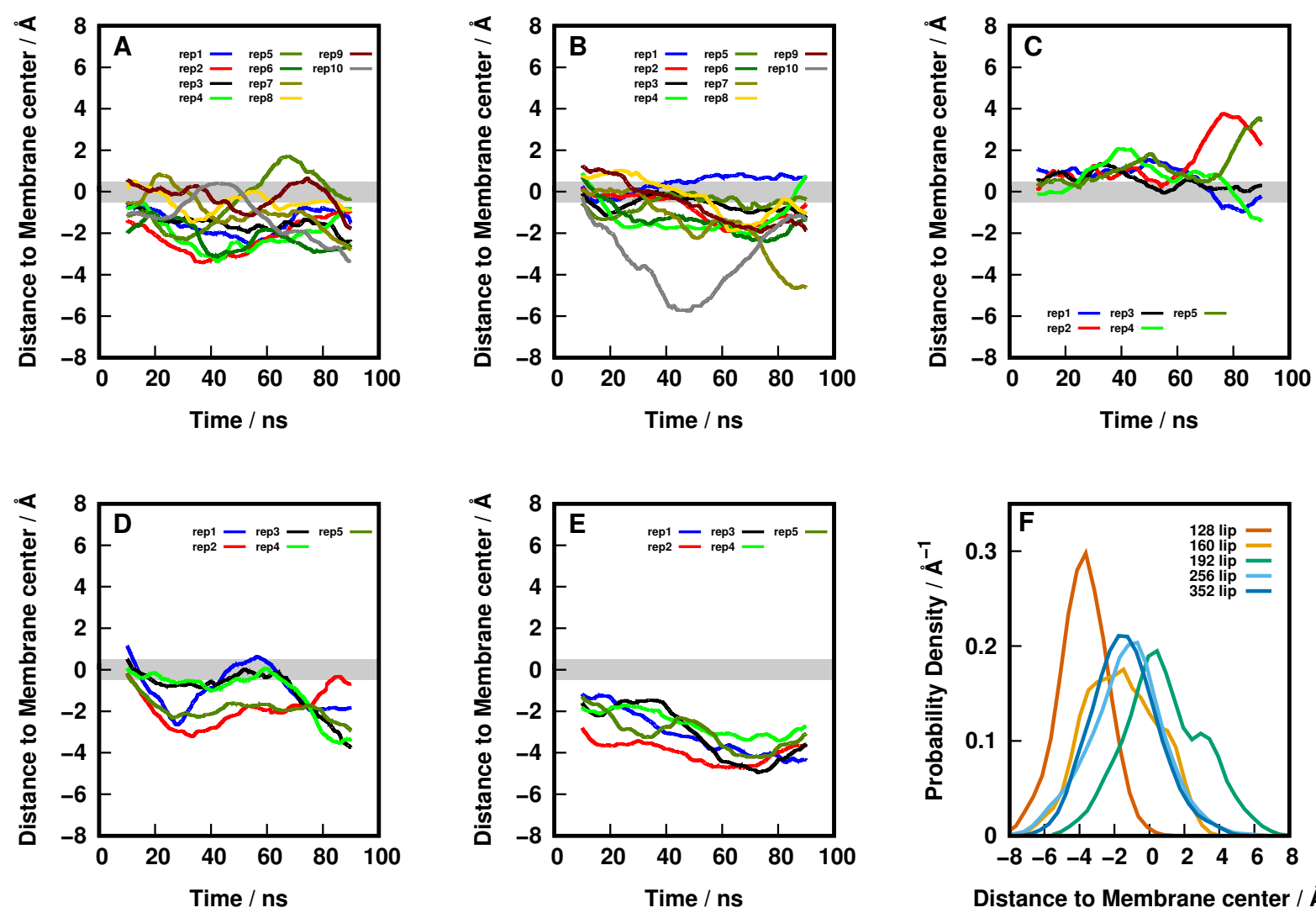

Figure S1: Time series plot of Asp25 residue distance relative to the membrane center for all simulated systems at pH 6.0 (352 lipids, A; 256 lipids, B; 192 lipids, C; 160 lipids, D; 128 lipids, E). This residue is known to sample the membrane center in unperturbed membrane systems. ${ }^{1}$ At $0 \AA$, the grey region represents the membrane center, while positive distance values indicate the residue is at the $\mathrm{N}$-terminus membrane monolayer, while negative distance values refer to the C-terminus monolayer. Probability density distribution calculated with the previous data for every lipid membrane system (F). The time series show the overall peptide vertical movement along the simulations and the distributions depict the average peptide vertical position using Asp25 as reference. 


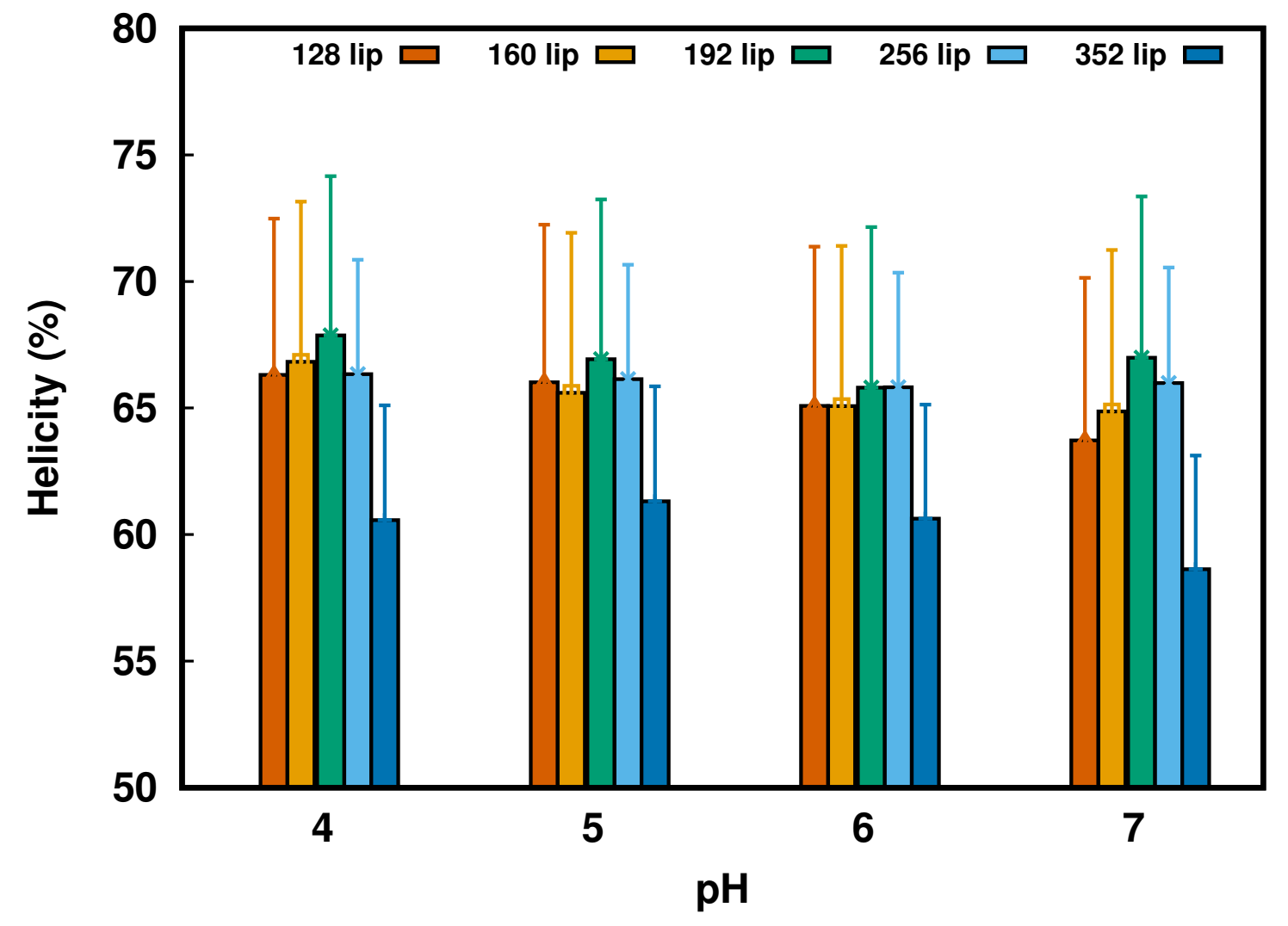

Figure S2: Average helicity percentage of wt-pHLIP in state III obtained through DSSP analysis, at each $\mathrm{pH}$ value, for every lipid membrane system. The represented helicity values are a measurement of peptide folding equilibrium in the sampled conformational space. The errors bar shown were obtained by calculating the standard error of the mean. 

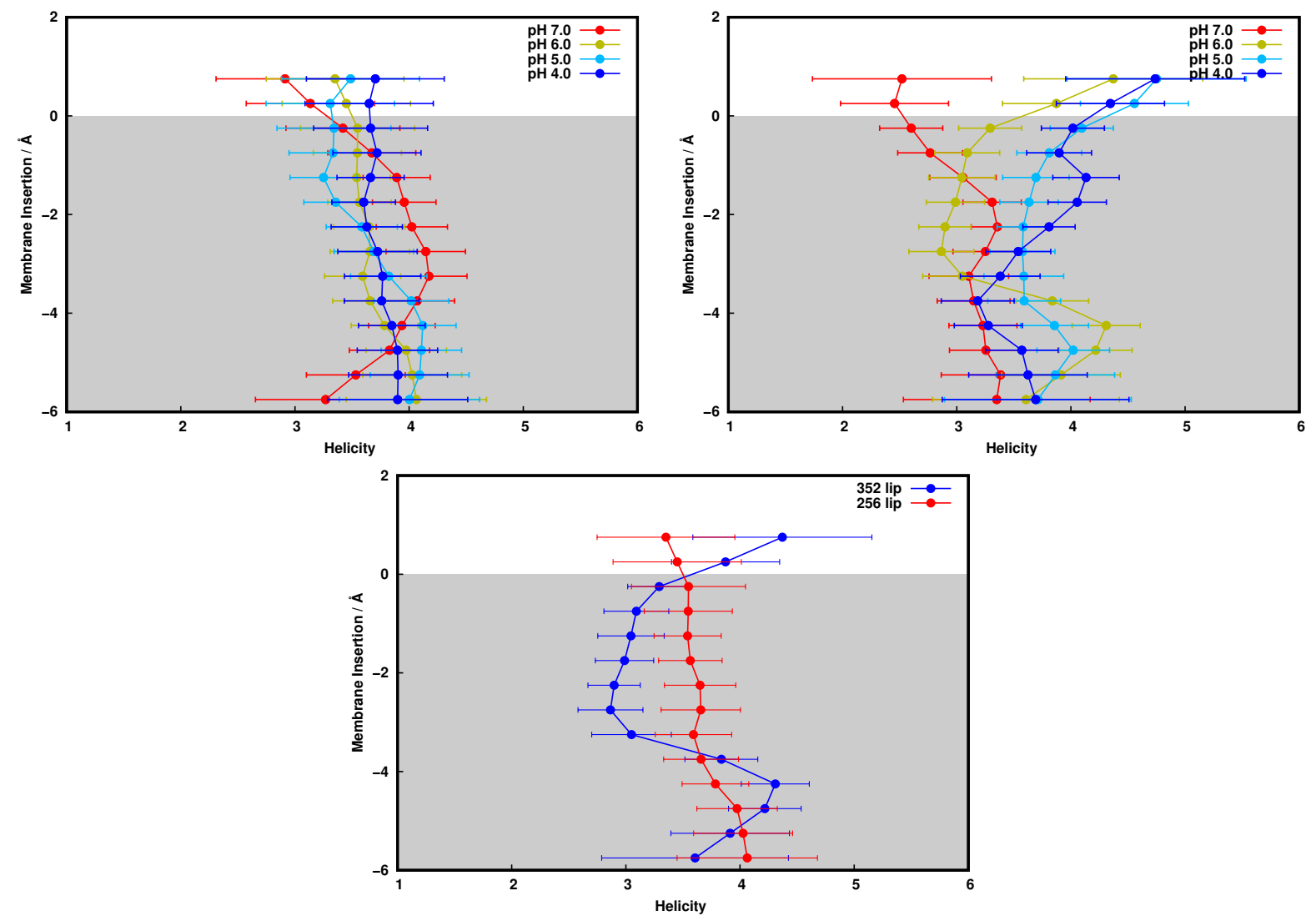

Figure S3: Helicity profiles for the Asp14 region of pHLIP (residues 12-16) at different pH values for the 256 (top left) and 352 (top right) lipid systems. A direct comparison between the two systems at $\mathrm{pH} 6.0$ is also shown (bottom plot). The helicity is quantified by counting the number of residues in helical secondary structure classifications from the DSSP criteria. $^{2}$ 


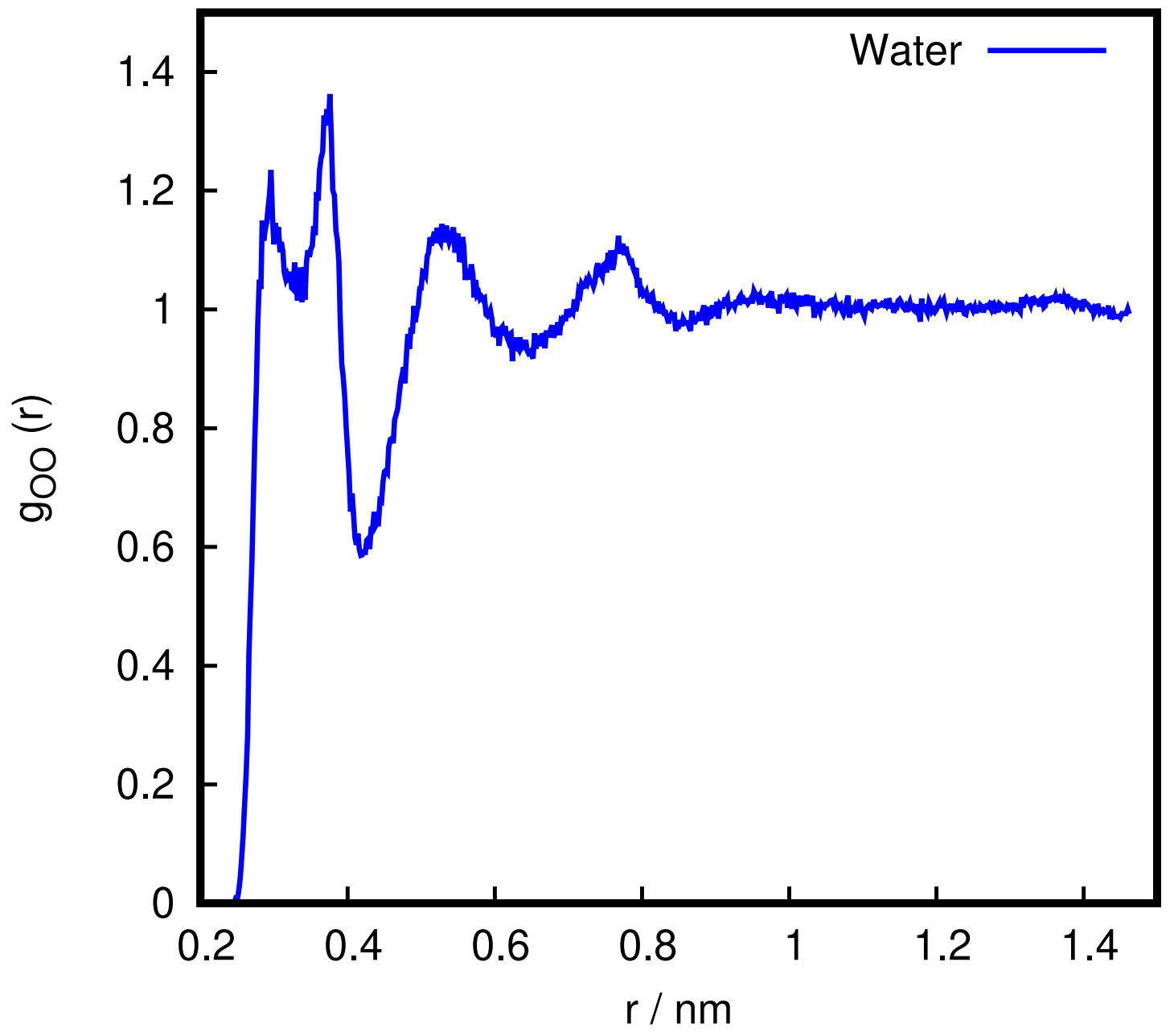

Figure S4: Water radial distribution function (RDF) from an aspartate pentapeptide in a 128 DMPC membrane bilayer. The data was extracted from previous published simulations. ${ }^{3}$ Both water oxygens were used for the calculations. The first, second, and third solvation shells are at $0.42,0.65$ and $0.85 \mathrm{~nm}$ respectively. The two peaks inside the first solvation shell appear due to established hydrogen bonds with either oxygen of the carboxylic group. 


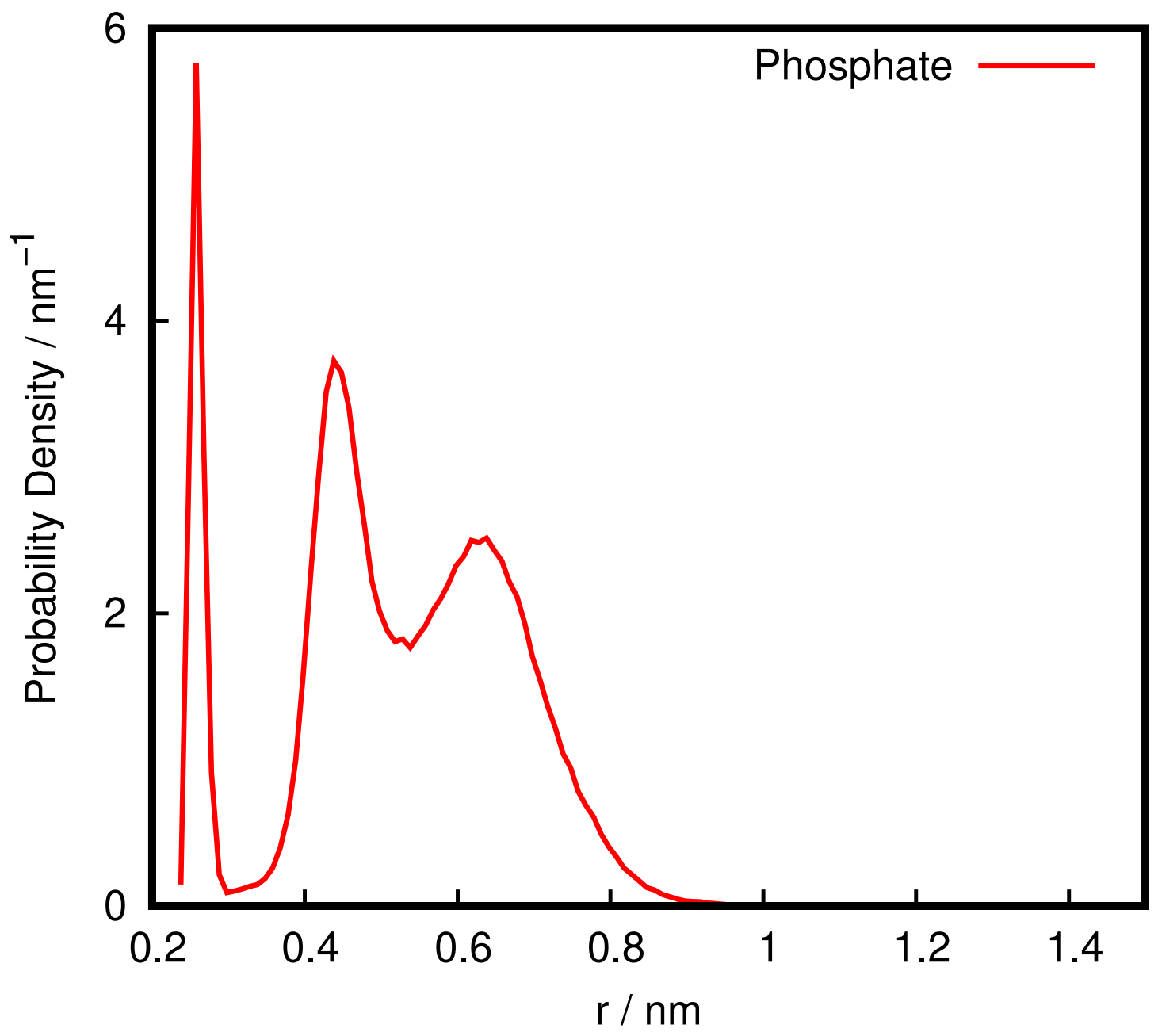

Figure S5: Minimum phosphate distance distributions for pHLIP Asp14 residue in the 256 POPC membrane bilayer. Both free oxygens of the phosphate group were used for the calculations. The interaction shell at $0.52 \mathrm{~nm}$ encapsulates all local interactions with the phosphate groups that compete with water molecules and include both direct and watermediated interactions. 


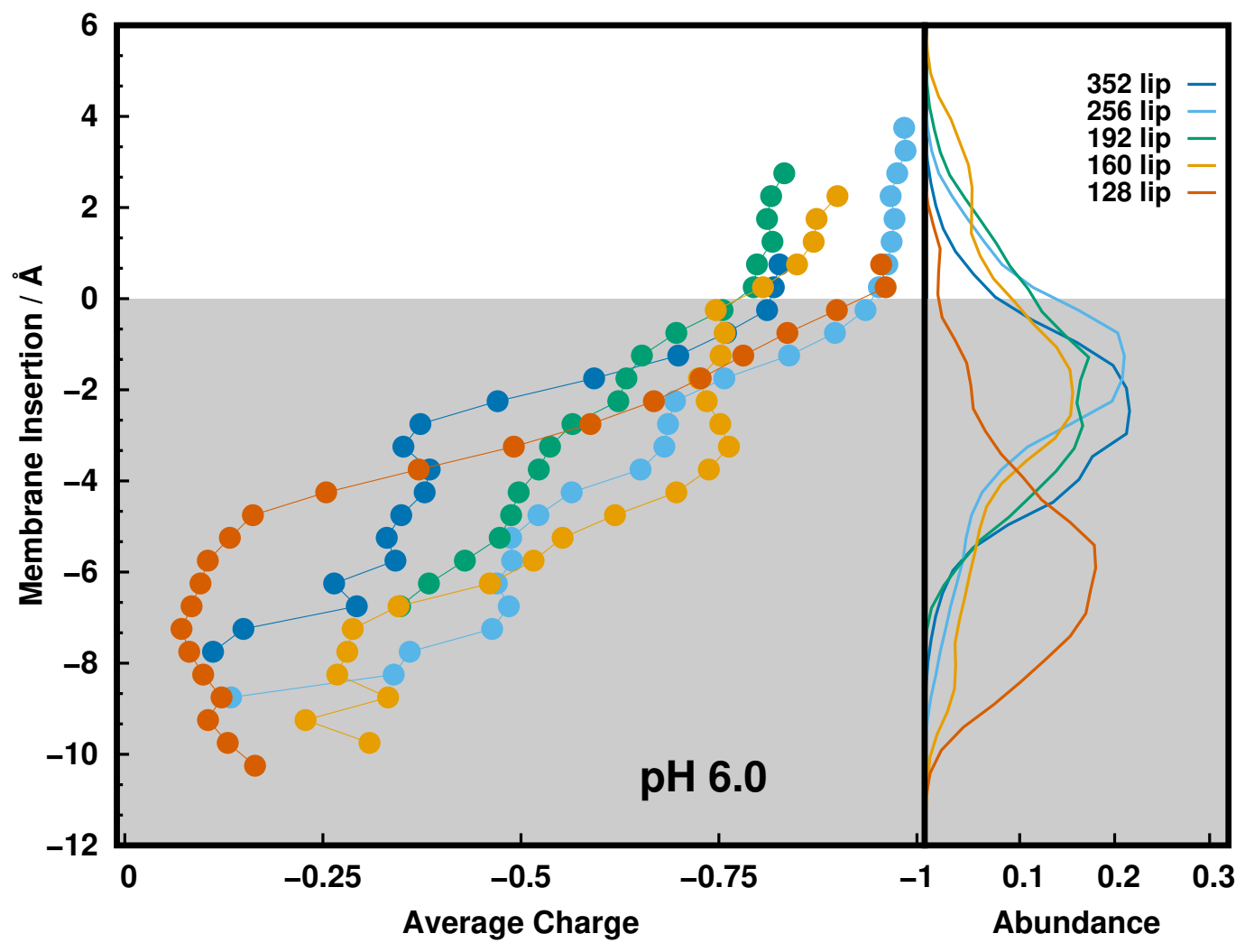

Figure S6: Protonation profiles and membrane insertion distributions of Asp14 at pH 6.0 for all simulated systems, including the one with 128 lipids. The membrane is highlighted using a grey-shaded region. The negative insertion values indicate residue insertion relative to the water/membrane interface defined by the phosphate groups $(0 \AA)$. The positive insertion values indicate that the residue is solvated by bulk water. 

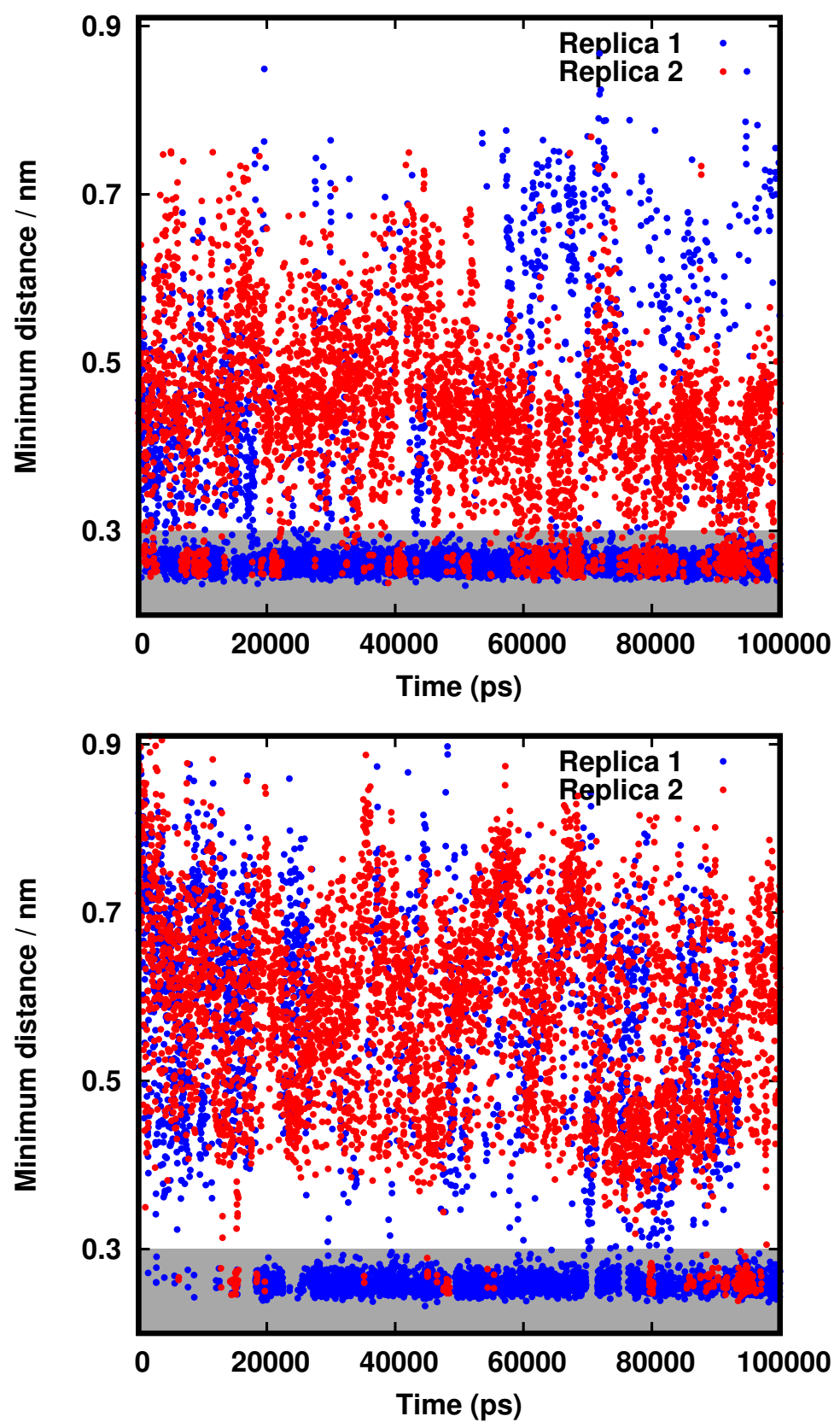

Figure S7: Time series plot of the minimum distance between the Asp14 carboxylic oxygen atoms and the free oxygen atoms of the phosphate groups. The data was obtained from two replicas of the 352 (upper plot) and 256 (bottom plot) lipid size system, illustrating two very distinct behaviours in our simulations. In replica 1 (blue dots), we observe a "lock" state behaviour, with Asp14 persistently interacting with a phosphate group most of the simulation time. Meanwhile, in replica 2 (red dots), the exhibited behaviour reveals an interchange between "on/off" states, where Asp14 is able to establish and break hydrogen bonds throughout the simulation. The grey shaded region highlights the range of distances (below $0.3 \mathrm{~nm}$ ) defining a stable hydrogen bond between Asp14 and a phosphate group. 
Table S1: The abundance and persistence of the hydrogen bonds between between the Asp14 carboxylic oxygen atoms and the free oxygen atoms of the phosphate groups in the two larger lipidic systems. The H-bond abundance was calculated over the complete equilibrated replicas in all replicates, using a cutoff of $0.3 \mathrm{~nm}$ to define a stable hydrogen bond. The Hbond persistence is the average length of all simulation segments where the hydrogen bond occurs.

\begin{tabular}{||ccc||}
\hline System & H-bond Abund. & H-bond persistence $(\mathrm{ps})$ \\
\hline \hline 256 & $21.4 \pm 6.6$ & $135.6 \pm 42.9$ \\
\hline 352 & $34.1 \pm 7.7$ & $205.0 \pm 60.2$ \\
\hline
\end{tabular}




\section{References}

(1) Vila-Viçosa, D.; Silva, T. F.; Slaybaugh, G.; Reshetnyak, Y. K.; Andreev, O. A.; Machuqueiro, M. Membrane-Induced $\mathrm{p} K_{\mathrm{a}}$ Shifts in wt-pHLIP and Its L16H Variant. J. Chem. Theory Comput. 2018, 14, 3289-3297.

(2) Kabsch, W.; Sander, C. Dictionary of Protein Secondary Structure: Pattern Recognition of Hydrogen-Bonded and Geometrical Features. Biopolymers 1983, 22, 2577-2637.

(3) Teixeira, V. H.; Vila-Viçosa, D.; Reis, P. B. P. S.; Machuqueiro, M. pKa Values of Titrable Amino Acids at the Water/Membrane Interface. J. Chem. Theory Comput. 2016, 12, 930-934. 